\title{
PNPRS OFFICERS AND MEMBERS 2019
}

\section{Officers}

1. Dr. Jove Jim S. Aguas (University of Santo Tomas, Manila) - Executive Governor

2. Dr. Juan Rafael G. Macaranas (De La Salle-College of Saint Benilde, Manila) - Chairman

3. Dr. Danilo S. Alterado (Saint Louis University, Baguio) - Vice Chairman

4. Dr Jeffrey L. Bartilet (Polytechnic University of the Philippines, Manila) - Secretary

5. Mr. Alvin O. Tan (University of Santo Tomas, Manila) - Treasurer

6. Dr. Fleurdeliz R. Altez-Albela (University of Santo Tomas, Manila) - Auditor

7. Dr. Rodrigo D. Abenes (Philippine Normal University, Manila) - Public Relations Officer

8. Ms. Ma. Glovedi Joy L. Bigornia (Hebei Foreign Studies University, Hebei, China) Legal Officer

9. Ms. Basilisa P. Macaranas - Administrative Officer

\section{Members}

10. Ms. Aireen T. Abiera (Marikina Polytechnic College, Marikina City)

11. Dr. Carmelito Nomer S. Abolencia (Eastern Visayas State University, Tacloban City)

12. Dr. Victor R. Aguilan (Silliman University, Dumaguete)

13. Ms. Ninotchka Mumtaj B. Albano (De La Salle University, Manila)

14. Dr. Albert C. Albina (Negros Oriental State University, Bayawan-Sta. Catalina)

15. Dr. Wilhelm Julian Alfredo (Department of Agrarian Reform, Quezon City)

16. Dr. Marian Ambrozy (College of International Business ISM in Presov, Slovakia)

17. Dr. Emmanuel Ifeanyi Ani (University of Ghana, Legon, Ghana)

18. Mr. Gilbert A. Apas (Pamantasan ng Lungsod ng Maynila, Manila)

19. Mr. Julius O. Basallo (Cebu Technological University-Barili Campus)

20. Mr. Dennis M. Bautista (Leyte Normal University, Tacloban City)

21. Mr. Henry P. Bilan (Kalinga State University, Kalinga)

22. Ms. Roxanne T. Bongco (Bataan Peninsula State University)

23. Mr. Elmer P. Brabante (Polytechnic University of the Philippines, Manila)

24. Dr. Mark Calano (Ateneo de Manila University, Quezon City)

25. Mr. Gian Edric Hilario G. Catambing (Cabantuan City)

26. Mr. Delfin P. Chiniona (Technological Institute of the Philippines)

27. Dr. Dalmacito A. Cordero, Jr. (De La Salle University, Manila)

28. Dr. Franz Giuseppe F. Cortez (University of Santo Tomas, Manila)

29. Mr. Jose Fermin B. Crave (Cebu Normal University, Cebu City) 
30. Dr. Robelyn P. Cunanan (Batangas State University-Main I, Batangas City)

31. Dr. Charlie M. Dagwasi (Benguet State University)

32. Mr. Zacarias G. Damo, Jr. (Mariano Marcos State University)

33. Mr. Roy John R. Del Rosario (Centro Escolar University, Manila)

34. Mr. Beljun P. Enaya (Visayas State University, Baybay City)

35. Dr. Edwin Etieyibo (University of the Witwatersrand, Johannesburg, South Africa)

36. Dr. Guiraldo C. Fernandez, Jr. (Visayas State University, Baybay City)

37. Dr. Crescencia C. Gabijan (Focolare Movement, Tagaytay City)

38. Mr. Prince Airick S. Gapo (Fiat Lux Academe-Cavite, Imus City)

39. Mr. Aldrin Mathew L. Go (Manila)

40. Dr. Eden M. Gripaldo (Retired-University of the Philippines-Diliman, Quezon City)

41. Ms. Veniz Maja V. Guzman(De La Salle University-Manila)

42. Mr. Aldrin S. Jaramilla (University of Northern Philippines, Vigan City)

43. Mr. Joseph Martin M. Jose (De La Salle University - Manila)

44. Dr. Danilo L. Libradilla (Cebu Technological University-Barili Campus, Cebu)

45. Dr. Leslie Anne L. Liwanag (Visayas State University, Baybay City)

46. Mr. Edward L. Macariola (Saint Louis University, Baguio City)

47. Mr. Erwin A. Mallo (University of Southern Mindanao, North Cotabato)

48. Dr. Rhochie Avelino E. Matienzo (University of Santo Tomas, Manila)

49. Ms. Meshille A. Mitra (Timoteo Paez Elementary School, Pasay City)

50. Dr. Robert A. Montaña (University of Santo Tomas, Manila)

51. Mr. Romeo F. Narvaez (Mindanao State University, General Santos City)

52. Dr. Ngoc Thi Minh Nguyen (Institute of Sociology, Hanoi, Vietnam)

53. Mr. Marc Benigno C. Olaguir (Bohol State University, Bohol)

54. Dr. Ian Raymond B. Pacquing (University of Santo Tomas, Manila)

55. Mr. Joseph P. Paña (De La Salle University, Manila)

56. Dr. Engelbert C. Pasag (Panpacific University, Urdaneta City)

57. Mr. Christopher A. Pelias (Far Eastern University - Manila)

58. Mr. John Paul J. Petrola (University of San Agustin, Iloilo City)

59. Dr. Joezenon A. Purog (Eastern Visayas State University, Tacloban City)

60. Mr. Beaujorne Sirad A. Ramirez (Immaculate Conception Academy, Greenhills)

61. Dr. Christine Carmela R. Ramos (Mapua University, Manila)

62. Mr. Virgilio A. Rivas (Polytechnic University of the Philippines, Manila)

63. Mr. Mark Louie Tabunan (University of Northern Philippines, Ilocos Sur)

64. Dr.Alex Y.Tiempo (Cebu Normal University, Cebu City)

65. Mr. Jose Z. Tria (Catanduanes State University)

66. Dr. Michal Valco (University of Presov, Slovakia)

67. Mr. Benito T. Villareal (Far Eastern University-Manila)

68. Mr. Al Franjon M. Villaroya (Visayas State University, Baybay City) 